Appl. Set-Valued Anal. Optim. 1 (2019), No. 1, pp. 53-62

Available online at http://asvao.biemdas.com

https://doi.org/10.23952/asvao.1.2019.1.05

\title{
LOCAL CONVERGENCE ANALYSIS OF JARRATT-TYPE SCHEMES FOR SOLVING EQUATIONS
}

\author{
IOANNIS K. ARGYROS ${ }^{1}$, SANTHOSH GEORGE ${ }^{2, *}$ \\ ${ }^{1}$ Department of Mathematical Sciences, Cameron University, Lawton, OK 73505, USA \\ ${ }^{2}$ Department of Mathematical and Computational Sciences, National Institute of Technology Karnataka 575 025, India
}

\begin{abstract}
The aim of this paper is to present a local convergence analysis for Jarratt-type schemes to solve equations with Banach space valued operators. The convergence conditions are are weaker than the corresponding ones in earlier results and some numerical examples are provided.
\end{abstract}

Keywords. Banach space; Jarratt-type scheme; Local convergence.

2010 Mathematics Subject Classification. 65H10, 65D10, 49M15.

\section{INTRODUCTION}

Let $\mathscr{E}_{1}, \mathscr{E}_{2}$ stand for Banach spaces and $\Omega \subseteq \mathscr{E}_{1}$ be a nonempty and open set. Let $\mathscr{E}_{1}: \Omega \longrightarrow \mathscr{E}_{2}$ be a continuously differentiable operator in the sense of Fréchet. The task of solving equation of the form

$$
F(x)=0
$$

is very important since problems from many disciplines can be reduced to (1.1); see, e.g., [1, 2, 3, 4, 5, 8, $13,21]$ and the references therein. A locally unique solution $x_{*}$ is desirable in a closed form but this can be achieved only in special cases. That explains why researchers and practitioners utilize some iterative schemes to approximate $x_{*}$. The most widely used schemes are the so called Newton-type.

Recently, there are many results, based on the Lipschitz-type conditions on the higher order derivatives, on local and semi-local convergence analysis of Newton-type schemes; see [1, 2, 3, 4, 5, 10, 11, $12,14,15,16,17,18,19,22]$ and the references therein. The convergence domain in these studies is small in general. These conditions limit the applicability of these method. As a motivational example, let us define a function $F$ on $\mathscr{E}_{1}=\left[-\frac{1}{2}, \frac{5}{2}\right]$ by

$$
F(x)=\left\{\begin{array}{l}
x^{3} \ln x^{2}+x^{5}-x^{4}, x \neq 0 \\
0, x=0
\end{array}\right.
$$

${ }^{*}$ Corresponding author.

E-mail addresses: iargyros@ cameron.edu (A. K. Argyros), sgeorge@ nitk.edu.in (S. George).

Received March 11, 2019; Accepted March 22, 2019.

(C)2019 Applied Set-Valued Analysis and Optimization 
Letting $x^{*}=1$, we have

$$
\begin{aligned}
F^{\prime}(x) & =3 x^{2} \ln x^{2}+5 x^{4}-4 x^{3}+2 x^{2}, F^{\prime}(1)=3, \\
F^{\prime \prime}(x) & =6 x \ln x^{2}+20 x^{3}-12 x^{2}+10 x \\
F^{\prime \prime \prime}(x) & =6 \ln x^{2}+60 x^{2}-24 x+22 .
\end{aligned}
$$

It is obvious that function $F$ does not have bounded third derivative in $\mathscr{E}_{1}$.

In this paper, we study the following Jarratt-type scheme defined by

$$
\begin{aligned}
y_{k} & =x_{k}-F^{\prime}\left(x_{k}\right)^{-1} F\left(x_{k}\right), \\
z_{k} & =x_{k}+\frac{2}{3}\left(y_{k}-x_{k}\right), \\
u_{k} & =x_{k}-A_{k} F^{\prime}\left(x_{k}\right)^{-1} F\left(x_{k}\right), \\
x_{k+1} & =u_{k}-\left[\frac{3}{2} F^{\prime}\left(z_{k}\right)^{-1} A_{k}+F^{\prime}\left(x_{k}\right)^{-1}\left(I-\frac{3}{2} A_{k}\right)\right] F\left(u_{k}\right), \\
A_{k} & =\left(6 F^{\prime}\left(z_{k}\right)-2 F^{\prime}\left(x_{k}\right)\right)^{-1}\left(3 F^{\prime}\left(z_{k}\right)+F^{\prime}\left(x_{k}\right)\right)
\end{aligned}
$$

for $n=0,1,2, \ldots$. Our local convergence analysis uses only hypotheses on the first Fréchet derivative, whereas the order of convergence is established using (COC) and (ACOC) (see Remark 2.1 (b)). Hence, we expand the applicability of method (1.2). The organization is as follows. In Section 2, we give the local convergence of method (1.2). In last section, Section 3, numerical examples are provided.

\section{LOCAL CONVERGENCE}

Let us consider some scalar functions and parameters which will appear in the local convergence analysis. Consider $w_{0}:[0,+\infty) \longrightarrow[0,+\infty)$ to be a continuous and increasing function with $w_{0}(0)=0$. Suppose that the equation

$$
w_{0}(t)=1
$$

has at least one positive solution. Denote by $\rho_{0}$ the smallest such solution. Let us also consider function $w:\left[0, \rho_{0}\right) \longrightarrow[0,+\infty)$ to be continuous and increasing with $w(0)=0$. Define functions $g_{1}, g_{2}, h_{1}$ and $h_{2}$ on the interval $\left[0, \rho_{0}\right)$ by

$$
\begin{gathered}
g_{1}(t)=\frac{\int_{0}^{1} w((1-\theta) t) d \theta}{1-w_{0}(t)}, \\
h_{1}(t)=g_{1}(t)-1, \\
g_{2}(t)=\frac{1}{3}\left(1+2 g_{1}(t)\right)
\end{gathered}
$$

and

$$
h_{2}(t)=g_{2}(t)-1 \text {. }
$$

We have $h_{1}(0)=-1$ and $h_{1}(t) \longrightarrow+\infty$ as $t \longrightarrow \rho_{0}^{-}$. The intermediate value theorem guarantees that equation $h_{1}(t)=0$ has at least one solution in $\left(0, \rho_{0}\right)$. Denote by $r_{1}$ the smallest such solution. We also have that $h_{2}(0)=-\frac{2}{3}$ and $h_{2}(t) \longrightarrow+\infty$ as $t \longrightarrow \rho_{0}^{-}$. Denote by $r_{2}$ the smallest solution of equation $h_{2}(t)=0$ in $\left(0, \rho_{0}\right)$. Suppose that equation

$$
p(t)=1
$$


has at least one solution in $\left(0, \rho_{0}\right)$, where $p(t)=\frac{1}{2}\left(3 w_{0}\left(g_{2}(t) t\right)+w_{0}(t)\right)$. Denote by $\rho_{1}$ the smallest such solution. Set $\rho_{2}=\min \left\{\rho_{0}, \rho_{1}\right\}$. Define functions $g_{3}$ and $h_{3}$ on the interval $\left[0, \rho_{2}\right)$ by

$$
g_{3}(t)=g_{1}(t)+\frac{3}{4} \frac{\left(w_{0}\left(g_{2}(t) t\right)+w_{0}(t)\right) \int_{0}^{1} v(\theta t) d \theta}{(1-p(t))\left(1-w_{0}(t)\right)}
$$

and

$$
h_{3}(t)=g_{3}(t)-1,
$$

where $v:\left[0, \rho_{2}\right) \longrightarrow[0,+\infty)$ is a continuous and increasing function. We get $h_{3}(0)=-1$ and $h_{3}(t) \longrightarrow$ $+\infty$ as $t \longrightarrow \rho_{2}^{-}$. Denote by $r_{3}$ the smallest solution of equation $h_{3}(t)=0$ in $\left(0, \rho_{2}\right)$. Suppose that equation

$$
w_{0}\left(g_{2}(t) t\right)=1
$$

and

$$
w_{0}\left(g_{3}(t) t\right)=1
$$

have at least one solution in $\left(0, \rho_{2}\right)$. Denote by $\rho_{3}$ and $\rho_{4}$, the smallest such solutions, respectively. Set $\rho=\min \left\{\rho_{3}, \rho_{4}\right\}$. Define functions $g_{4}$ and $h_{4}$ on $(0, \rho)$ by

$$
\begin{aligned}
g_{4}(t)= & {\left[\frac{\int_{0}^{1} w\left((1-\theta) g_{3}(t) t\right) d \theta}{1-w_{0}\left(g_{3}(t) t\right)}\right.} \\
& +\frac{\left(w_{0}\left(g_{3}(t) t\right)+w_{0}(t)\right) \int_{0}^{1} v\left(\theta g_{3}(t) t\right) d \theta}{\left(1-w_{0}\left(g_{3}(t) t\right)\right)\left(1-w_{0}(t)\right)} \\
& \left.+\frac{3}{8} \frac{\left(3 v\left(g_{2}(t) t\right)+v(t)\right)\left(w_{0}\left(g_{2}(t) t\right)+w_{0}(t)\right) \int_{0}^{1} v\left(\theta g_{3}(t) t\right) d \theta}{\left(1-w_{0}\left(g_{2}(t) t\right)\right)\left(1-w_{0}(t)\right)(1-p(t))}\right] g_{3}(t)
\end{aligned}
$$

and

$$
h_{4}(t)=g_{4}(t)-1 \text {. }
$$

We obtain $h_{4}(0)=-1$ and $h_{4}(t) \longrightarrow+\infty$ as $t \longrightarrow \rho^{-}$. Denote by $r_{4}$ the smallest solution of equation $h_{4}(t)=0$ in $(0, \rho)$. Moreover, define the radius of convergence $r$ by

$$
r=\min \left\{r_{i}\right\}, i=1,2,3,4 \text {. }
$$

Then, we have that, for each $t \in[0, r)$,

$$
\begin{aligned}
& 0 \leq w_{0}(t)<1 \\
& 0 \leq p(t)<1, \\
& 0 \leq w_{0}\left(g_{2}(t) t\right)<1, \\
& 0 \leq w_{0}\left(g_{3}(t) t\right)<1
\end{aligned}
$$

and

$$
0 \leq g_{i}(t)<1, i=1,2,3,4 .
$$

Let $B(q, a), \bar{B}(q, a)$ denote the open and closed balls in $\mathscr{E}_{1}$, respectively, with center $q \in \mathscr{E}_{1}$ and radius $a>0$. We utilize in the local convergence analysis the proceeding notation and the conditions $(\mathrm{C})$ :

(c1) $F: \Omega \subset \mathscr{E}_{1} \longrightarrow \mathscr{E}_{2}$ is a continuously differentiable operator in the sense of Fréchet and there exists $x_{*} \in \Omega$ such that $F\left(x_{*}\right)=0$ and $F^{\prime}\left(x_{*}\right)^{-1} \in \mathscr{L}\left(\mathscr{E}_{2}, \mathscr{E}_{1}\right)$. 
(c2) There exists function $w_{0}:[0,+\infty) \longrightarrow[0,+\infty)$ continuous and increasing with $w_{0}(0)=0$ such that, for each $x \in \Omega$,

$$
\left\|F^{\prime}\left(x_{*}\right)^{-1}\left(F^{\prime}(x)-F^{\prime}\left(x_{*}\right)\right)\right\| \leq w_{0}\left(\left\|x-x_{*}\right\|\right)
$$

Set $\Omega_{0}=\Omega \cap B\left(x_{*}, \rho_{0}\right)$, where $\rho_{0}$ is defined in (2.1).

(c3) There exist functions $w:\left[0, \rho_{0}\right) \longrightarrow[0,+\infty), v:\left[0, \rho_{0}\right) \longrightarrow[0,+\infty)$ with $w(0)=0$ such that, for each $x, y \in \Omega_{0}$,

$$
\left\|F^{\prime}\left(x_{*}\right)^{-1}(F(y)-F(x))\right\| w(\|y-x\|)
$$

and

$$
\left\|F^{\prime}\left(x_{*}\right)^{-1} F^{\prime}(x)\right\| \leq v\left(\left\|x-x_{*}\right\|\right) .
$$

(c4) $\bar{B}\left(x_{*}, r\right) \subseteq \Omega$, where $r$ is defined in (2.5).

(c5) There exists $r_{*} \geq r$ such that

$$
\int_{0}^{1} w_{0}\left(\theta r_{*}\right) d \theta<1
$$

Set $\Omega_{1}=\Omega \cap \bar{U}\left(x_{*}, r_{*}\right)$.

Next, we present the local convergence analysis of method (1.2).

Theorem 2.1. Suppose that conditions $(C)$ hold. Then, the sequence $\left\{x_{k}\right\}$ starting at $x_{0} \in B\left(x_{*}, r\right)-\left\{x_{*}\right\}$ and generated by method (1.2) is well defined, remains in $B\left(x_{*}, r\right)$ for each $k=0,1,2, \ldots$ and converges to $x_{*}$ so that

$$
\begin{aligned}
\left\|y_{k}-x_{*}\right\| & \leq g_{1}\left(\left\|x_{k}-x_{*}\right\|\right)\left\|x_{k}-x_{*}\right\| \leq\left\|x_{k}-x_{*}\right\|<r, \\
\left\|z_{k}-x_{*}\right\| & \leq g_{2}\left(\left\|x_{k}-x_{*}\right\|\right)\left\|x_{k}-x_{*}\right\| \leq\left\|x_{k}-x_{*}\right\|, \\
\left\|u_{k}-x_{*}\right\| & \leq g_{3}\left(\left\|x_{k}-x_{*}\right\|\right)\left\|x_{k}-x_{*}\right\| \leq\left\|x_{k}-x_{*}\right\|
\end{aligned}
$$

and

$$
\left\|x_{k+1}-x_{*}\right\| \leq g_{4}\left(\left\|x_{k}-x_{*}\right\|\right)\left\|x_{k}-x_{*}\right\| \leq\left\|x_{k}-x_{*}\right\|,
$$

where the functions $g_{i}$ are defined as previous. Moreover, the limit point $x_{*}$ is the only solution of the equation $F(x)=0$ in $\Omega_{1}$ defined in (c5).

Proof. Mathematical induction is used to prove estimates (2.11)-(2.14). Let $x \in B\left(x_{*}, r\right)-\left\{x_{*}\right\}$. By (2.1), (2.5) and (c2), it follows that

$$
\left\|F^{\prime}\left(x_{*}\right)^{-1}\left(F^{\prime}(x)-F^{\prime}\left(x_{*}\right)\right)\right\| \leq w_{0}\left(\left\|x-x_{*}\right\|\right) \leq w_{0}(r)<1,
$$

which along the Banach perturbation Lemma [3] shows that $F^{\prime}(x)^{-1} \in \mathscr{L}\left(\mathscr{E}_{2}, \mathscr{E}_{1}\right)$ and

$$
\left\|F^{\prime}(x)^{-1} F^{\prime}\left(x_{*}\right)\right\| \leq \frac{1}{1-w_{0}\left(\left\|x-x_{*}\right\|\right)} .
$$


We also have that $y_{0}$ and $z_{0}$ are well defined by the first and second substep of method (1.2) for $n=0$. By (2.5), (2.10) (for $i=1,2)$, (c1), the first condition in (c3) and (2.15), we have that

$$
\begin{aligned}
\left\|y_{0}-x_{*}\right\| & =\left\|x_{0}-x_{*}-F^{\prime}\left(x_{0}\right)^{-1} F\left(x_{0}\right)\right\| \\
& \leq\left\|F^{\prime}\left(x_{0}\right)^{-1} F^{\prime}\left(x_{*}\right)\right\|\left\|\int_{0}^{1} F^{\prime}\left(x_{*}\right)^{-1}\left(F^{\prime}\left(x_{*}+\theta\left(x_{0}-x_{*}\right)\right)-F^{\prime}\left(x_{0}\right)\right)\left(x_{0}-x_{*}\right) d \theta\right\| \\
& \leq \frac{\int_{0}^{1} w\left((1-\theta)\left\|x_{0}-x_{*}\right\|\right) d \theta}{1-w_{0}\left(\left\|x_{0}-x_{*}\right\|\right)}\left\|x_{0}-x_{*}\right\| \\
& \leq g_{1}\left(\left\|x_{0}-x_{*}\right\|\right)\left\|x_{0}-x_{*}\right\| \leq\left\|x_{0}-x_{*}\right\|<r .
\end{aligned}
$$

Hence, (2.11) holds for $n=0$, and $y_{0} \in B\left(x_{*}, r\right)$. Moreover, using (2.16), we have

$$
\begin{aligned}
\left\|z_{0}-z_{*}\right\| & =\left\|x_{0}-x_{*}+\frac{2}{3}\left[\left(y_{0}-x_{*}\right)+\left(x_{*}-x_{0}\right)\right]\right\| \\
& =\frac{1}{3}\left\|x_{0}-x_{*}+2\left(y_{0}-x_{*}\right)\right\| \\
& \leq \frac{1}{3}\left(\left\|x_{0}-x_{*}\right\|+2\left\|y_{0}-x_{*}\right\|\right) \\
& \leq \frac{1}{3}\left(1+2 g_{1}\left(\left\|x_{0}-x_{*}\right\|\right)\right)\left\|x_{0}-x_{*}\right\| \\
& =g_{2}\left(\left\|x_{0}-x_{*}\right\|\right)\left\|x_{0}-x_{*}\right\| \leq\left\|x_{0}-x_{*}\right\|<r .
\end{aligned}
$$

So, (2.1) holds for $n=0$ and $z_{0} \in B\left(x_{*}, r\right)$. We show that $A_{0}$ is well defined. Using (2.5), (2.9), (c2), (2.16) and (2.17), we get that

$$
\begin{aligned}
& \|\left(2 F^{\prime}\left(x_{*}\right)\right)^{-1}\left[3 F^{\prime}\left(z_{0}\right)-F^{\prime}\left(x_{0}\right)-2 F^{\prime}\left(x_{*}\right)\right] \\
\leq & \frac{1}{2}\left[3\left\|F^{\prime}\left(x_{*}\right)^{-1}\left(F^{\prime}\left(z_{0}\right)-F^{\prime}\left(x_{*}\right)\right)\right\|+\left\|F^{\prime}\left(x_{*}\right)^{-1}\left(F^{\prime}\left(x_{0}\right)-F^{\prime}\left(x_{*}\right)\right)\right\|\right] \\
\leq & \frac{1}{2}\left(3 w_{0}\left(\left\|z_{0}-x_{*}\right\|\right)+w_{0}\left(\left\|x_{0}-x_{*}\right\|\right)\right) \\
\leq & \frac{1}{2}\left(3 w_{0}\left(g_{2}\left(\left\|x_{0}-x_{*}\right\|\right)\left\|x_{0}-x_{*}\right\|\right)+w_{0}\left(\left\|x_{0}-x_{*}\right\|\right)\right) \\
= & p\left(\left\|x_{0}-x_{*}\right\|\right) \leq p(r)<1 .
\end{aligned}
$$

It follows that $\left(3 F^{\prime}\left(z_{0}\right)-F^{\prime}\left(x_{0}\right)\right)^{-1} \in \mathscr{L}\left(\mathscr{E}_{2}, \mathscr{E}_{1}\right), A_{0}$ is well defined and

$$
\left\|\left(3 F^{\prime}\left(z_{0}\right)-F^{\prime}\left(x_{0}\right)\right)^{-1} F^{\prime}\left(x_{*}\right)\right\| \leq \frac{1}{2\left(1-p\left(\left\|x_{0}-x_{*}\right\|\right)\right)} .
$$

We can write

$$
\begin{aligned}
I-A_{0} & =\left(6 F^{\prime}\left(z_{0}\right)-2 F^{\prime}\left(x_{0}\right)\right)^{-1}\left[6 F^{\prime}\left(z_{0}\right)-2 F^{\prime}\left(x_{0}\right)-\left(3 F^{\prime}\left(x_{0}\right)+F^{\prime}\left(x_{0}\right)\right)\right] \\
& =\frac{3}{2}\left(3 F^{\prime}\left(z_{0}\right)-F^{\prime}\left(x_{0}\right)\right)^{-1}\left[\left(F^{\prime}\left(z_{0}\right)-F^{\prime}\left(x_{*}\right)\right)+\left(F^{\prime}\left(x_{*}\right)-F^{\prime}\left(x_{0}\right)\right)\right],
\end{aligned}
$$

which shows that

$$
\left\|I-A_{0}\right\| \leq \frac{3}{4} \frac{w_{0}\left(\left\|z_{0}-x_{*}\right\|\right)+w_{0}\left(\left\|x_{0}-x_{*}\right\|\right)}{1-p\left(\left\|x_{0}-x_{*}\right\|\right)} .
$$

Then, $u_{0}$ is well defined by the third substep of method (1.2) for $n=0$ and

$$
u_{0}-x_{*}=\left(x_{0}-x_{*}-F^{\prime}\left(x_{0}\right)^{-1} F\left(x_{0}\right)\right)+\left(I-A_{0}\right) F^{\prime}\left(x_{0}\right)^{-1} F^{\prime}\left(x_{*}\right) F^{\prime}\left(x_{*}\right)^{-1} F\left(x_{0}\right) .
$$


We can write by (c1) that

$$
F(x)=F(x)-F\left(x_{*}\right)=\int_{0}^{1} F^{\prime}\left(x_{*}+\theta\left(x-x_{*}\right)\right) d \theta\left(x-x_{*}\right) .
$$

From the second condition in (c3), we have

$$
\left\|F^{\prime}\left(x_{*}\right)^{-1} F(x)\right\| \leq \int_{0}^{1} v\left(\theta\left\|x-x_{*}\right\|\right) d \theta\left\|x-x_{*}\right\|
$$

since $\left\|x_{*}+\theta\left(x-x_{*}\right)-x_{*}\right\|=\theta\left\|x-x_{*}\right\|<r$ (i.e., $x_{*}+\theta\left(x-x_{*}\right) \in B\left(x_{*}, r\right)$ for each $\left.\theta \in[0,1]\right)$.

To continue, use (2.5), (2.10) (for $i=3),(2.15)-(2.18)$ and (2.20)-(2.22) to get that

$$
\begin{aligned}
\left\|u_{0}-x_{*}\right\| \leq & \left\|x_{0}-x_{*}-F^{\prime}\left(x_{0}\right)^{-1} F\left(x_{0}\right)\right\| \\
& +\left\|I-A_{0}\right\|\left\|F^{\prime}\left(x_{0}\right)^{-1} F\left(x_{*}\right)\right\|\left\|F^{\prime}\left(x_{*}\right)^{-1} F\left(x_{0}\right)\right\| \\
\leq & g_{1}\left(\left\|x_{0}-x_{*}\right\|\right)\left\|x_{0}-x_{*}\right\| \\
& +\frac{3}{4} \frac{\left(w_{0}\left(\left\|z_{0}-x_{*}\right\|\right)+w_{0}\left(\left\|x_{0}-x_{*}\right\|\right)\right) \int_{0}^{1} v\left(\theta\left\|x_{0}-x_{*}\right\|\right) d \theta}{\left(1-p\left(\left\|x_{0}-x_{*}\right\|\right)\right)\left(1-w_{0}\left(\left\|x_{0}-x_{*}\right\|\right)\right)}\left\|x_{0}-x_{*}\right\| \\
= & g_{3}\left(\left\|x_{0}-x_{*}\right\|\right)\left\|x_{0}-x_{*}\right\| \leq\left\|x_{0}-x_{*}\right\|<r .
\end{aligned}
$$

Hence, (2.13) holds for $n=0$ and $u_{0} \in B\left(x_{*}, r\right)$. Since $z_{0}, u_{0} \in B\left(x_{*}, r\right)$, we have as in (2.15) for $x=z_{0}, u_{0}$ that $F^{\prime}\left(z_{0}\right)^{-1} \in \mathscr{L}\left(\mathscr{E}_{2}, \mathscr{E}_{1}\right), F^{\prime}\left(u_{0}\right)^{-1} \in \mathscr{L}\left(\mathscr{E}_{2}, \mathscr{E}_{1}\right)$,

$$
\begin{aligned}
& \left\|F^{\prime}\left(z_{0}\right)^{-1} F^{\prime}\left(x_{*}\right)\right\| \leq \frac{1}{1-w_{0}\left(\left\|z_{0}-x_{*}\right\|\right)}, \\
& \left\|F^{\prime}\left(u_{0}\right)^{-1} F^{\prime}\left(x_{*}\right)\right\| \leq \frac{1}{1-w_{0}\left(\left\|u_{0}-x_{*}\right\|\right)}
\end{aligned}
$$

and $x_{1}$ is well defined by the last substep of method (1.2). Moreover, we can write

$$
\begin{aligned}
x_{1}-x_{*}= & u_{0}-x_{*}-F^{\prime}\left(x_{0}\right)^{-1} F\left(u_{0}\right) \\
& -\frac{3}{2}\left(F^{\prime}\left(z_{0}\right)^{-1}-F^{\prime}\left(x_{0}\right)^{-1}\right) A_{0} F\left(u_{0}\right) \\
= & \left(u_{0}-x_{*}-F^{\prime}\left(u_{0}\right)^{-1} F\left(u_{0}\right)\right)+F^{\prime}\left(u_{0}\right)^{-1}\left[\left(F^{\prime}\left(x_{0}\right)-F^{\prime}\left(x_{*}\right)\right)\right. \\
& \left.+\left(F^{\prime}\left(x_{*}\right)-F^{\prime}\left(u_{0}\right)\right)\right] F^{\prime}\left(x_{0}\right)^{-1} F\left(u_{0}\right) \\
& -\frac{3}{2} F^{\prime}\left(z_{0}\right)^{-1}\left[\left(F^{\prime}\left(x_{0}\right)-F^{\prime}\left(x_{*}\right)\right)\right. \\
& \left.+\left(F^{\prime}\left(x_{*}\right)-F^{\prime}\left(z_{0}\right)\right)\right] F^{\prime}\left(x_{0}\right)^{-1} A_{0} F\left(u_{0}\right) .
\end{aligned}
$$


Using (2.5), (2.10) (for $i=4),(2.15),(2.22)$ (for $x=u_{0}$ ) and (2.23)- (2.27), and the triangle inequality, we obtain that

$$
\begin{aligned}
\left\|x_{1}-x_{*}\right\| \leq & \left\|u_{0}-x_{*}-F^{\prime}\left(x_{0}\right)^{-1} F\left(u_{0}\right)\right\| \\
& +\left\|F^{\prime}\left(u_{0}\right)^{-1} F^{\prime}\left(x_{*}\right)\right\|\left[\left\|F^{\prime}\left(x_{*}\right)^{-1}\left(F^{\prime}\left(x_{0}\right)-F^{\prime}\left(x_{*}\right)\right)\right\|\right. \\
& \left.+\left\|F^{\prime}\left(x_{*}\right)^{-1}\left(F^{\prime}\left(x_{*}\right)-F^{\prime}\left(u_{0}\right)\right)\right\|\right]\left\|F^{\prime}\left(x_{0}\right)^{-1} F^{\prime}\left(x_{*}\right)\right\|\left\|F^{\prime}\left(x_{*}\right)^{-1} F\left(u_{0}\right)\right\| \\
& +\frac{3}{2}\left\|F^{\prime}\left(z_{0}\right)^{-1} F^{\prime}\left(x_{*}\right)\right\|\left[\left\|F^{\prime}\left(x_{*}\right)^{-1}\left(F^{\prime}\left(x_{0}\right)-F^{\prime}\left(x_{*}\right)\right)\right\|\right. \\
& \left.+\left\|F^{\prime}\left(x_{*}\right)^{-1}\left(F^{\prime}\left(x_{*}\right)-F^{\prime}\left(z_{0}\right)\right)\right\|\right] \\
& \times\left\|F^{\prime}\left(x_{0}\right)^{-1} F^{\prime}\left(x_{*}\right)\right\|\left\|F^{\prime}\left(x_{*}\right)^{-1} A_{0}\right\| F^{\prime}\left(x_{*}\right)^{-1} F\left(u_{0}\right) \| \\
\leq & \frac{\int_{0}^{1} w\left((1-\theta)\left\|u_{0}-x_{*}\right\|\right) d \theta\left\|u_{0}-x_{*}\right\|}{1-w_{0}\left(\left\|u_{0}-x_{*}\right\|\right)} \\
& +\frac{\left(w_{0}\left(\left\|u_{0}-x_{*}\right\|\right)+w_{0}\left(\left\|x_{0}-x_{*}\right\|\right)\right) \int_{0}^{1} v\left(\theta\left\|u_{0}-x_{*}\right\|\right) d \theta\left\|u_{0}-x_{*}\right\|}{\left(1-w_{0}\left(\left\|u_{0}-x_{*}\right\|\right)\right)\left(1-w_{0}\left(\left\|x_{0}-x_{*}\right\|\right)\right)} \\
& +\frac{3}{8} \frac{\left(3 v\left(\left\|z_{0}-x_{*}\right\|\right)+v\left(\left\|x_{0}-x_{*}\right\|\right)\right)\left(w_{0}\left(\left\|z_{0}-x_{*}\right\|\right)+w_{0}\left(\left\|x_{0}-x_{*}\right\|\right)\right)}{\left(1-w_{0}\left(\left\|z_{0}-x_{*}\right\|\right)\right)\left(1-w_{0}\left(\left\|x_{0}-x_{*}\right\|\right)\right)\left(1-p\left(\left\|x_{0}-x_{*}\right\|\right)\right)} \\
& \times \int_{0}^{1} v\left(\theta\left\|u_{0}-x_{*}\right\|\right) d \theta\left\|u_{0}-x_{*}\right\| \\
\leq & g_{4}\left(\left\|x_{0}-x_{*}\right\|\right)\left\|x_{0}-x_{*}\right\| \leq\left\|x_{0}-x_{*}\right\|<r .
\end{aligned}
$$

So, (2.14) holds for $n=0$ and $x_{1} \in B\left(x_{*}, r\right)$. If $x_{i}, y_{i}, z_{i}, u_{i}, x_{i+1}$ replace $x_{0}, y_{0}, z_{0}, u_{0}, x_{1}$ in the preceding estimates, we complete the induction for (2.11)-(2.14). Then, it follows from the estimate

$$
\left\|x_{i+1}-x_{*}\right\| \leq c\left\|x_{i}-x_{*}\right\|<r
$$

where $c=g_{4}\left(\left\|x_{0}-x_{*}\right\|\right) \in[0,1)$, that $\lim _{i \longrightarrow+\infty} x_{i}=x_{*}$ and $x_{i+1} \in B\left(x_{*}, r\right)$. Let

$$
Q=\int_{0}^{1} F^{\prime}\left(x_{*}+\theta\left(y_{*}-x_{*}\right)\right) d \theta
$$

for some $y_{*} \in \Omega_{1}$ with $F\left(y_{*}\right)=0$. In view of (c2) and (c5), we get that

$$
\begin{aligned}
\left\|F^{\prime}\left(x_{*}\right)^{-1}\left(Q-F^{\prime}\left(x_{*}\right)\right)\right\| & \leq \int_{0}^{1} w_{0}\left(\theta\left\|y_{*}-x_{*}\right\|\right) d \theta \\
& \leq \int_{0}^{1} w_{0}\left(\theta r_{*}\right) d \theta<1,
\end{aligned}
$$

so $Q^{-1} \in \mathscr{L}\left(\mathscr{E}_{2}, \mathscr{E}_{1}\right)$. Finally, in view of

$$
0=F\left(y_{*}\right)-F\left(x_{*}\right)=Q\left(y_{*}-x_{*}\right),
$$

we deduce that $x_{*}=y_{*}$. This completes the proof.

Remark 2.1. (a) Let $w_{0}(t)=L_{0} t, w(t)=L t$. The radius $\tilde{\rho}_{1}=\frac{2}{2 L_{0}+L}$ was obtained by Argyros as the convergence radius for Newton's method under condition (c1)-(c3) [1, 2, 3]. Notice that the convergence radius for Newton's method given independently by Rheinboldt [17] and Traub [21] was given by

$$
\tilde{\rho}=\frac{2}{3 L}<\tilde{\rho}_{1}
$$


Let $f(x)=e^{x}-1$. Then $x^{*}=0$. Setting $\Omega=B(0,1)$,we have that $L_{0}=e-1<L=e^{\frac{1}{L_{0}}}$. So $\tilde{\rho}=0.24252961<\tilde{\rho}_{1}=0.3827$.

Moreover, the new error bounds $[17,21]$ are:

$$
\left\|x_{n+1}-x^{*}\right\| \leq \frac{L}{1-L_{0}\left\|x_{n}-x^{*}\right\|}\left\|x_{n}-x^{*}\right\|^{2},
$$

whereas the old ones [20, 21]

$$
\left\|x_{n+1}-x^{*}\right\| \leq \frac{L}{1-L\left\|x_{n}-x^{*}\right\|}\left\|x_{n}-x^{*}\right\|^{2} .
$$

Clearly, the new error bounds are more precise if $L_{0}<L$. Clearly, the radius of convergence of method (1.2) given by $r$ is smaller than $\tilde{\rho}_{1}$.

(b) We can use the computational order of convergence (COC) [23]

$$
\xi=\frac{\ln \frac{\left\|x_{n+2}-x_{n+1}\right\|}{\left\|x_{n+1}-x_{n}\right\|}}{\ln \frac{\left\|x_{n+1}-x_{n}\right\|}{\left\|x_{n}-x_{n-1}\right\|}}, \quad \text { for each } n=1,2, \ldots
$$

or the approximate computational order of convergence (ACOC) [23]

$$
\xi^{*}=\frac{\ln \frac{\left\|x_{n+2}-x^{*}\right\|}{\left\|x_{n+1}-x^{*}\right\|}}{\ln \frac{\left\|x_{n+1}-x^{*}\right\|}{\left\|x_{n}-x^{*}\right\|}}, \quad \text { for each } n=0,1,2, \ldots
$$

(c) Using (h2) and

$$
\begin{aligned}
\left\|G^{\prime}\left(x^{*}\right)^{-1} G^{\prime}(x)\right\| & =\left\|G^{\prime}\left(x^{*}\right)^{-1}\left(G^{\prime}(x)-G^{\prime}\left(x^{*}\right)\right)+I\right\| \\
& \leq 1+\left\|G^{\prime}\left(x^{*}\right)^{-1}\left(G^{\prime}(x)-G^{\prime}\left(x^{*}\right)\right)\right\| \leq 1+q_{0}\left(\left\|x-x^{*}\right\|\right),
\end{aligned}
$$

the second condition in (h3) can be replaced by

$$
w(t)=1+w_{0}(t)
$$

or

$$
w(t)=1+w_{0}\left(\rho_{0}\right) .
$$

Notice that if, in particular, $w_{0}(t)=L_{0} t$, then, we can choose $w(t)=2$, since $t \in\left[0, \frac{1}{L_{0}}\right)$.

\section{NUMERICAL EXAMPLES}

Example 3.1. Let $\mathscr{E}_{1}=\mathscr{E}_{2}=\mathbb{R}^{3}, \Omega=\bar{U}(0,1), x^{*}=(0,0,0)^{T}$. Define function $F$ on $\Omega$ for $w=(x, y, z)^{T}$ by

$$
F(w)=\left(e^{x}-1, \frac{e-1}{2} y^{2}+y, z\right)^{T} .
$$

Then, the Fréchet-derivative is defined by

$$
F^{\prime}(v)=\left[\begin{array}{ccc}
e^{x} & 0 & 0 \\
0 & (e-1) y+1 & 0 \\
0 & 0 & 1
\end{array}\right]
$$

We have $w_{0}(t)=(e-1) t, w(t)=e^{\frac{1}{e-1}} t$ and $v(t)=e^{\frac{1}{e-1}}$.

Therefore, we have

$$
\begin{gathered}
r_{1}=r_{2}=0.38269191223238574472986783803208, \\
r_{3}=0.36509279210603506893306757774553
\end{gathered}
$$


and

$$
r_{4}=r=0.22889602845498427385884099294344 \text {. }
$$

Example 3.2. Returning back to the motivational example at the introduction of this paper, we have $w_{0}(t)=w(t)=L_{0} t$ and $v(t)=2$ where $L_{0}=96.662907$. Then, the radius are:

$$
\begin{gathered}
r_{2}=r_{2}=0.0068968199628702108600064590859802, \\
r_{3}=0.0068968199628702099926447210975766
\end{gathered}
$$

and

$$
r_{4}=r=0.002743265590198637297880690510965 \text {. }
$$

Example 3.3. Let $\mathscr{E}_{1}=\mathscr{E}_{2}=C[0,1], \Omega=\bar{U}\left(x^{*}, 1\right)$ and consider the nonlinear integral equation of the mixed Hammerstein-type $[1,2,6,7,8,9,12]$ defined by

$$
x(s)=\int_{0}^{1} G(s, t)\left(x(t)^{3 / 2}+\frac{x(t)^{2}}{2}\right) d t,
$$

where the kernel $G$ is the Green's function defined on the interval $[0,1] \times[0,1]$ by

$$
G(s, t)= \begin{cases}(1-s) t, & t \leq s \\ s(1-t), & s \leq t\end{cases}
$$

The solution $x^{*}(s)=0$ is the same as the solution of equation $(1.1)$, where $\left.F: C[0,1] \longrightarrow C[0,1]\right)$ is defined by

$$
F(x)(s)=x(s)-\int_{0}^{1} G(s, t)\left(x(t)^{3 / 2}+\frac{x(t)^{2}}{2}\right) d t .
$$

Since

$$
\left\|\int_{0}^{1} G(s, t) d t\right\| \leq \frac{1}{8}
$$

we have

$$
F^{\prime}(x) y(s)=y(s)-\int_{0}^{1} G(s, t)\left(\frac{3}{2} x(t)^{1 / 2}+x(t)\right) d t .
$$

From $F^{\prime}\left(x^{*}(s)\right)=I$, we have

$$
\left\|F^{\prime}\left(x^{*}\right)^{-1}\left(F^{\prime}(x)-F^{\prime}(y)\right)\right\| \leq \frac{1}{8}\left(\frac{3}{2}\|x-y\|^{1 / 2}+\|x-y\|\right) .
$$

It follows that $w_{0}(t)=w(t)=\frac{1}{8}\left(\frac{3}{2} t^{1 / 2}+t\right), v(t)=1+w_{0}(t)$. Hence

$$
r_{1}=r_{2}=r_{3}=2.1333333333333333333333333333333
$$

and

$$
r_{4}=1.342649884740344745637230516877 \text {. }
$$

So we choose $r=1$. 


\section{REFERENCES}

[1] I.K. Argyros, Computational theory of iterative methods, Series: Studies in Computational Mathematics, 15, Editors: C.K. Chui and L. Wuytack, Elsevier, New York, 2007.

[2] I.K. Argyros, Convergence and applications of Newton-type iterations, Springer Verlag, New York, 2008.

[3] I. K. Argyros, A. A. Magreñañ, Iterative Methods and their dynamics with applications: A Contemporary Study, CRC Press, 2017.

[4] R Behl, A. Cordero, S. S. Motsa, J. R. Torregrosa, Stable high order iterative methods for solving nonlinear models, Appl. Math. Comput. 303 (2017), 70-88.

[5] A. Cordero, J. R. Torregrosa, M. P. Vassileva, Pseudocomposition: a technique to design predictor-corrector methods for systems of nonlinear equations, Appl. Math. Comput. 218 (2012), 11496-11504.

[6] K. Madru, J. Jayaraman, Some higher order Newton-like methods for solving system of nonlinear equations and its applications, Int. J. Appl. Comput. Math. 3 (2017), 2213-2230.

[7] J. A. Ezquerro, M.A. Hernández, Recurrence relations for Chebyshev-type methods, Appl. Math. Optim. 41 (2000), $227-$ 236.

[8] J. A. Ezquerro, M.A. Hernández, Multipoint super-Halley type approximation algorithms in Banach spaces, Numer. Funct. Anal. Optimiz. 21 (2000), 845-858.

[9] J. A. Ezquerro, M.A. Hernández, A modification of the super-Halley method under mild differentiability condition, J. Comput. Appl. Math. 114 (2000), 405-409.

[10] J. A. Ezquerro, M.A. Hernández, A generalization of the Kantorovich type assumptions for Halley's method, Int. J. Comput. Math. 84 (2007), 1771-1779.

[11] M. Grau-Sanchez, A. Grau, M. Noguera, Ostrowski type methods for solving systems of nonlinear equations, Appl. Math. Comput. 218 (2011), 2377-2385.

[12] J. M. Gutiérrez, A.A. Magreñán and N. Romero, On the semi-local convergence of Newton-Kantorovich method under center-Lipschitz conditions, Appl. Math. Comput. 221 (2013), 79-88.

[13] L.V. Kantorovich, G.P. Akilov, Functional Analysis, Pergamon Press, Oxford, 1982.

[14] A. A. Magreńãn, Different anomalies in a Jarratt family of iterative root finding methods, Appl. Math. Comput. 233 (2014), 29-38.

[15] A. A. Magreńãn, A new tool to study real dynamics: The convergence plane, Appl. Math. Comput. 248 (2014), 29-38.

[16] F. A. Potra, V. Pták, Nondiscrete Induction and Iterative Processes, in: Research Notes in Mathematics, Vol. 103, Pitman, Boston, 1984.

[17] W. C. Rheinboldt, An adaptive continuation process for solving systems of nonlinear equations, Pol. Acad. Sci. (Banach Ctr. Publ.) 3 (1978), 129-142.

[18] J.R. Sharma, H. Arora, On efficient weighted-Newton methods for solving system of nonlinear equations, Appl. Math. Comput. 222 (2013), 497-506.

[19] J.R. Sharma, H. Arora, Efficient Jarratt-like methods for solving systems of nonlinear equations, Calcolo, 51 (2014), 193-210.

[20] J.R. Sharma, P. Gupta, An efficient fifth order method for solving systems of nonlinear equations, Comput. Math. Appl. 67 (2014), 591-601.

[21] J.F. Traub, Iterative methods for the solution of equations, Prentice Hall Englewood Cliffs, New Jersey, USA, 1964.

[22] X. Wang, J. Kou, Semilocal convergence of a modified muli-point Jarratt method in Banach spaces under general continuity condition, Numer. Algorithms 60 (2012), 369-390.

[23] S. Weerakoon, T.G.I. Fernando, A variant of Newton's method with accelerated third-order convergence, Appl. Math. Lett. 13 (2000), 87-93. 